gerungen dieser Arbeit auf die Sozialwissenschaften im Allgemeinen übertragen lassen, muss im Einzelfall geprüft werden.

\title{
1.4 ARGUMENTATIONSGANG DER ARBEIT
}

Für eine erste Annäherung wird die Thematik historisch eingeführt. Dies veranschaulicht, dass die Debatten um die Rolle der Soziologie in der Gesellschaft und um ihr Verhältnis zur Gesellschaft keineswegs neu sind. Dazu werden vier Zeitabschnitte betrachtet, in denen jeweils unterschiedliche Grenzziehungsaspekte der Soziologie im Mittelpunkt stehen (2.1). Die Schlussfolgerungen aus diesem Abschnitt zeigen, dass sich um das Verhältnis der Soziologie zu ihrem Untersuchungsgegenstand diverse Semantiken gebildet haben, die sich in Form verschiedener Dichotomisierungen um Grenzziehungen des Verhältnisses der Soziologie zu ihrem Untersuchungsgegenstand gruppieren.

In Kapitel 2.2 wird ein soziologischer Zugang entwickelt. Dabei werden gleichzeitig zwei Perspektivierungen der Untersuchung von Grenzen in der Soziologie geschaffen: Mithilfe der Systemtheorie ist der Blick von „innen“ auf die Soziologie mit dem Vokabular der Soziologie selbst möglich. Im Zentrum steht hier die Verhandlung der Grenzen der Soziologie, die über semantische Sinnerzeugung geschieht. Diese fertigt das System als sogenannte Selbstbeschreibungen mithilfe von Reflexionstheorien an. Dennoch ergeben sich bei der Übernahme dieser Perspektive mindestens zwei Schwierigkeiten. Die erste bezieht sich auf die Selbstreferenz und die ihr inhärente Schwierigkeit, eine Außenperspektive auf die Systemtheorie einzunehmen. Die nächste Schwierigkeit besteht darin, dass die Systemtheorie prinzipiell keine Akteure berücksichtigt.

Die zweite Perspektivierung beschäftigt sich mit dem Blick auf die Soziologie im Sinne einer Außenposition, die auch den Blick auf den Akteur zulässt. Dies erfolgt mit dem sozialkonstruktivistischen Konzept der boundary work (Gieryn, 1983). Im Zentrum stehen sogenannte ,ideologische Selbstbeschreibungen“, die sprachlich die Grenzen zwischen Soziologie und Untersuchungsgegenstand markieren. Obwohl die beiden Perspektivierungen von unterschiedlichen epistemologischen Annahmen ausgehen, finden sich Konvergenzen in der Rolle der Sprache in Hinblick auf Grenzkonstruktionen. Zudem lässt diese fruchtbare Verbindung der Ansätze zu, auf die epistemologischen Herausforderungen und die in 1.3 genannten Gefahren zu reagieren: Sie gewährleistet einen theoretischen Zugang zur Analyse von Grenzen in der Soziologie, der weder zirkulär noch relativistisch ist. 
In einem Fazit zu den theoretischen Konsequenzen (2.3) wird die bereits genannte These der Doppelstruktur begrifflich ausgearbeitet, epistemologisch spezifiziert und als „doppelte Konstitution der Soziologie“ markiert. Die methodologischen Konsequenzen formulieren die Bedingungen einer Untersuchung der Soziologie. Denn die letztlich gegebene doppelte Reflexivität dieser Arbeit spiegelt sich auch in der Frage einer soziologischen Analyse der Soziologie wider.

Deshalb liegt im methodischen Teil (3) ein besonderes Augenmerk nicht nur auf der adäquaten Auswahl der Untersuchungsmethode, sondern auch auf dem Sampling. Dazu wird auf zwei unterschiedliche methodologische Stränge zurückgegriffen: die Grounded Theory und die dokumentarische Methode nach Bohnsack, die sich komplementär zueinander verhalten. So bietet die Grounded Theory insbesondere die Möglichkeit, das theoretische Sampling der Soziologie zu reflektieren. Denn die Auswahl der Interviewten ist vor dem Hintergrund der Soziologie als einer stark ausdifferenzierten Disziplin besonders wichtig. Die dokumentarische Methode wiederum findet in der Auswertung der Interviews Anwendung. Über diese Methode ist es möglich, vergleichend-hermeneutisch zu analysieren, wie Soziologinnen und Soziologen Grenzen zu ihrem Untersuchungsgegenstand konstruieren (3.1). Empirisch analysiert werden die Interviews in zwei Abschnitten: das Verhältnis zur Soziologie (4.1) und das Verhältnis zwischen Soziologie und Untersuchungsgegenstand (4.2) betreffend. Ein Zwischenfazit (4.3) reflektiert zunächst die relevanten Ergebnisse der Analyse, während Abschnitt 5 und 6 Grenzziehungen der Soziologie zu ihrem Untersuchungsgegenstand in einen größeren Reflexionskontext stellt - in Hinblick auf eine Grundlegung zu einer Wissenschaftssoziologie der Soziologie - und eine Reformulierung des Verhältnisses der Soziologie zu ihrem Untersuchungsgegenstand anbietet. 